\title{
On the microscopic origins of relaxation processes in aqueous peptide solutions undergoing a glass transition
}

Cite as: J. Chem. Phys. 152, 234503 (2020); https://doi.org/10.1063/5.0010312

Submitted: 09 April 2020. Accepted: 22 May 2020. Published Online: 15 June 2020

M. Weigler, I. Combarro-Palacios, (D) S. Cerveny, and (D) M. Vogel
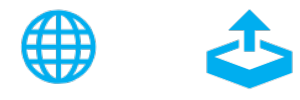

\section{ARTICLES YOU MAY BE INTERESTED IN}

Effect of heavy impurities on the dynamics of supercooled liquids

The Journal of Chemical Physics 152, 234502 (2020); https://doi.org/10.1063/5.0012096

Solvation structure and dynamics of the dimethylammonium cation diluted in liquid water: A molecular dynamics approach

The Journal of Chemical Physics 152, 234501 (2020); https://doi.org/10.1063/5.0004204

Water dynamics in the hydration shells of biological and non-biological polymers

The Journal of Chemical Physics 150, 234904 (2019); https://doi.org/10.1063/1.5096392

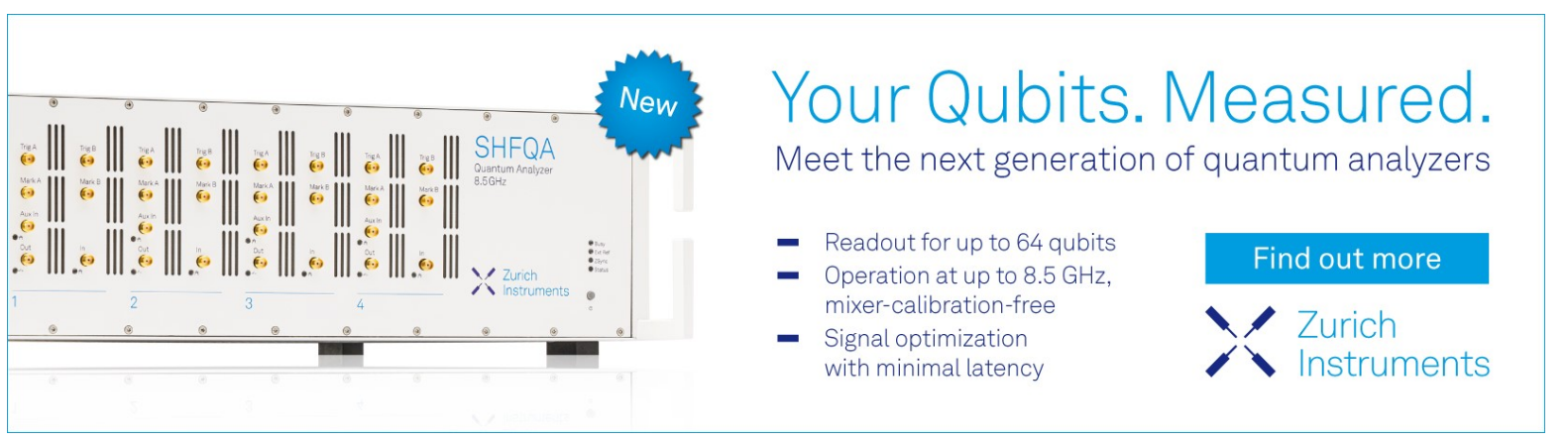




\title{
On the microscopic origins of relaxation processes in aqueous peptide solutions undergoing a glass transition
}

Cite as: J. Chem. Phys. 152, 234503 (2020); doi: 10.1063/5.0010312

Submitted: 9 April 2020 - Accepted: 22 May 2020 •

Published Online: 15 June 2020

M. Weigler, ${ }^{1}$ I. Combarro-Palacios, ${ }^{2}$ S. Cerveny, ${ }^{2,3}$ (D) and M. Vogel ${ }^{1, a)}$ (D)

\begin{abstract}
AFFILIATIONS
${ }^{1}$ Institut für Festkörperphysik, Technische Universität Darmstadt, Hochschulstr. 6, 64289 Darmstadt, Germany

${ }^{2}$ Centro de Fisica Materiales (CSIC-UPV/EHU) - Material Physics Centre (MPC), Paseo Manuel de Lardizabal 5, 20018 San Sebastian, Spain

${ }^{3}$ Donostia International Physics Center (DIPC), San Sebastian, Spain
\end{abstract}

a) Author to whom correspondence should be addressed: michael.vogel@physik.tu-darmstadt.de

\begin{abstract}
We combine broadband dielectric spectroscopy (BDS) with ${ }^{1} \mathrm{H}$ and ${ }^{2} \mathrm{H}$ nuclear magnetic resonance (NMR) to study molecular dynamics in mixtures of $\mathcal{\varepsilon}$-polylysine with $\mathrm{H}_{2} \mathrm{O}$ or $\mathrm{D}_{2} \mathrm{O}$. In $\mathrm{BDS}$, four relaxation processes can be attributed to molecular dynamics. While the fastest process $\mathrm{P} 1$ obeys the Arrhenius law, the slowest process $\mathrm{P} 4$ shows prominent non-Arrhenius behavior typical of structural $\alpha$ relaxation. For the intermediate processes $\mathrm{P} 2$ and $\mathrm{P} 3$, the temperature dependence changes at the glass transition temperature $T_{\mathrm{g}}$. The ${ }^{1} \mathrm{H}$ and ${ }^{2} \mathrm{H}$ NMR results yield insights into the molecular origins of these relaxation phenomena. In these NMR analyses, we exploit, in addition to the isotope selectivity of the method, the possibility to distinguish between various types of motion based on their respective line-shape effects and the capability to single out specific molecular moieties based on different spin-lattice relaxation behaviors. In this way, we reveal that process P1 results from the rotation of side and end groups of the peptide, while process P2 is caused by a reorientation of essentially all water molecules, which are quasi-isotropic and survive well below $T_{\mathrm{g}}$. As for the peptide backbone dynamics, we find evidence that rotational motion of polar groups is involved in process P3 and that nonpolar regions show a dynamical process, which is located between P3 and P4. Thus, the NMR analyses do not yield evidence for coexisting fast peptide-decoupled and slow peptide-coupled water species, which contribute to BDS processes P2 and $\mathrm{P} 3$, respectively, but minor bimodality of water motion may remain undetected. Finally, it is demonstrated that the proton/deuteron exchange needs to be considered when interpreting experimental results for molecular dynamics in aqueous peptide solutions.
\end{abstract}

Published under license by AIP Publishing. https://doi.org/10.1063/5.0010312

\section{INTRODUCTION}

Aqueous solutions of biological molecules are truly omnipresent. They show manifold phenomena, which have high relevance for biological functions and technological processes. ${ }^{1,2}$ Therefore, many scientific studies ascertained their thermodynamic features as well as their structural and dynamical behaviors. ${ }^{3-6}$ It is, meanwhile, well established that the properties of biomolecules critically depend on the existence of a hydration shell. ${ }^{7-10}$ In turn, the characteristics of water in these hydration shells usually differ from those in the bulk liquid. ${ }^{5,11,12}$ However, the detailed nature of biomolecule-water interactions is still elusive. ${ }^{7,13,14}$
Previous approaches to this research field often focused on the very important case of hydrated proteins. It was argued that water slaves ${ }^{7}$ or plasticizes ${ }^{13}$ protein dynamics. Regardless of the exact nature of the interplay, the importance of protein-solvent couplings is demonstrated by the finding that, unlike dry proteins, hydrated proteins start to show molecular motions related to biological functions when the temperature is increased above $\sim 200-240 \mathrm{~K} .^{7,13-15}$ Likewise, it was observed that water structure and dynamics are altered at protein surfaces; in particular, water in one or two hydration layers around the protein does not freeze, but undergoes glassy arrest upon cooling. ${ }^{16-19}$ 
Several studies strove for a common description of dynamics in various types of aqueous solutions. ${ }^{5,11,15,20-23}$ Mostly focusing on glass-forming mixtures, studies using broadband dielectric spectroscopy (BDS) revealed very complex dynamical behaviors with several coexisting relaxation processes, which were assigned to either of the components or to coupled motions of the constituents. It was reported that depending on the solute, but for both biological and nonbiological solutions, the plasticizing or slaving scenarios may apply. ${ }^{22}$ Specifically, the coupling scenario was proposed to depend on whether one or two independent water relaxations exist in the mixtures. ${ }^{23}$ In these approaches, it was proved particularly fruitful that peptide-water mixtures ideally bridge the gap between solutions of complex proteins and simple molecules.

Recent contributions exploited that $\varepsilon$-L-polylysine ( $\varepsilon$-PLL) is highly soluble in water when the chain length is moderate and reported that crystallization is suppressed over wide concentration and temperature ranges. ${ }^{22-24}$ Altogether, four dielectric processes related to molecular dynamics were found, where the fastest one (P1) is weak. Using information about the concentration dependence of the respective relaxation strengths and relaxation times, two intermediate dielectric processes ( $\mathrm{P} 2$ and $\mathrm{P} 3$ ) were attributed to water reorientations, whereas the slowest one (P4) was assigned to peptide reorientation. ${ }^{22-24}$ Specifically, P2 and P3 were found to grow with increasing water content and related to solute-decoupled and solute-coupled water dynamics, respectively, while $\mathrm{P} 4$ characterizes peptide dynamics involved in the glass transition of the mixtures, as inferred from comparison with results from differential scanning calorimetry.

Nuclear magnetic resonance (NMR) spectroscopy provides access to not only the rates but also the mechanisms of molecular motions in an isotope selective manner. ${ }^{25,26}$ Hence, this method is an ideal tool for studies of complex dynamics in aqueous peptide solutions. Specifically, when comparing results for various probe nuclei, it is possible to determine which components of the mixtures are involved in a particular dynamical process. Furthermore, previous NMR works on complex dynamics, ${ }^{27,28}$ including studies on hydrated proteins, ${ }^{29-32}$ revealed that broad dynamic ranges can be covered and, hence, the glassy slowdown can be followed by combining spin-lattice relaxation (SLR) experiments, line-shape analysis (LSA), and stimulated-echo (STE) measurements.

Here, we combine BDS and NMR studies to unravel the nature of molecular dynamics in glass-forming aqueous $\varepsilon$-PLL solutions. This combination of methods proved advantageous in several previous approaches to complex molecular dynamics in aqueous mixtures. $^{33-37}$ While BDS yields correlation times of various relaxation processes in broad temperature and dynamic ranges, ${ }^{1} \mathrm{H}$ and ${ }^{2} \mathrm{H}$ NMR on mixtures of $\varepsilon$-PLL with $\mathrm{H}_{2} \mathrm{O}$ and $\mathrm{D}_{2} \mathrm{O}$, respectively, provide information about their microscopic origins and, in particular, about the involved molecular moieties and the underlying motional mechanisms.

\section{METHODS}

We characterize molecular dynamics in the studied peptide solutions based on the rotational autocorrelation functions,

$$
F_{l}(t)=\frac{\left\langle P_{l}(\cos \theta(0)) P_{l}(\cos \theta(t))\right\rangle}{\left\langle P_{l}(\cos \theta(0)) P_{l}(\cos \theta(0))\right\rangle} .
$$

Here, $P_{l}$ denotes the Legendre polynomial of rank $l, \theta$ is an angle describing the molecular orientation, and the pointed brackets mark an ensemble average.

\section{A. Analysis of dielectric loss spectra}

In BDS, we utilize that, assuming linear response and neglecting intermolecular correlations, the dielectric permittivity $\varepsilon^{*}(\omega)$ is related to the correlation function $F_{1}(t)$, which describes the rotational motion of the dipole moments in the sample. To analyze $\varepsilon^{*}(\omega)$, we simultaneously fit the real and imaginary parts to a conductivity term and a superposition of Cole-Cole (CC) functions,

$$
\varepsilon^{*}(\omega)=\varepsilon_{\infty}+\frac{\sigma_{\mathrm{dc}}}{i \varepsilon_{0} \omega}+\sum_{n=1}^{N} \frac{\Delta \varepsilon_{n}}{1+\left(i \omega \tau_{n}\right)^{\alpha_{n}}} .
$$

Here, $\varepsilon_{\infty}$ is the high-frequency permittivity, $\varepsilon_{0}$ is the vacuum permittivity, and $\sigma_{\mathrm{dc}}$ denotes the dc conductivity. The relaxation processes are characterized by their respective relaxation strengths $\Delta \varepsilon_{n}$, relaxation times $\tau_{n}$, and width parameters $\alpha_{n}$. Further details of our fitting approach can be found in previous work. ${ }^{22}$

\section{B. Analysis of ${ }^{1} \mathrm{H}$ and ${ }^{2} \mathrm{H}$ data}

The present ${ }^{1} \mathrm{H}$ and ${ }^{2} \mathrm{H}$ NMR experiments probe the dipolar and quadrupolar interactions of the observed nuclei, respectively. Due to the anisotropic nature of these interactions, the ${ }^{1} \mathrm{H}$ and ${ }^{2} \mathrm{H}$ NMR frequencies $\omega$ depend on the molecular orientations, and hence, their time dependencies yield insights into molecular reorientations. In the following experiments, both water and peptide will contribute to the observed signals if no specific measures are taken, as will be discussed in some detail below.

${ }^{1} \mathrm{H}$ and ${ }^{2} \mathrm{H}$ LSA yield valuable information about both the rates and mechanisms for rotational motions. In our case, broad spectra result from the powder average over the anisotropic interactions when molecular dynamics is negligible at low temperatures. In more detail, broad Gaussian and Pake line shapes are observed in ${ }^{1} \mathrm{H}$ and ${ }^{2} \mathrm{H}$ NMR, respectively. By contrast, narrower spectra are found when rotational motion is sufficiently fast to average the dipolar and quadrupolar interactions at high temperatures. This line-shape transition occurs when the correlation time $\tau$ of molecular reorientation is of the order of the inverse spectral width in the static limit and, hence, in the microseconds regime. ${ }^{38,39}$ The geometry of the reorientation process determines the shape of the motionally averaged spectrum. While fast isotropic motion results in very narrow Lorentzian lines, fast anisotropic motion leaves a significant residual line width, which is determined by the geometry of the motion.

The line-shape transition is accompanied by minimum efficiency of echo pulse sequences used to record the NMR spectra because echo formation is hampered when molecular dynamics causes fluctuations of NMR frequencies $\omega$ during the defocussing and refocussing periods of the measurements. Hence, information about correlation times is available from analysis of the temperaturedependent spin echo intensity (SEI). In ${ }^{1} \mathrm{H}$ NMR, the magic sandwich echo or the time-reversal echo (TREV) sequences are commonly applied to revert the time evolution of the dipolar-coupled spin system. ${ }^{40,41}$ These echo sequences perform well at high and low temperatures, where the value of $\omega$ is unchanged, as averaged and 
static frequencies result from fast and slow dynamics, respectively. However, the performance breaks down when molecular dynamics on the time scale of the echo delay $\Delta_{e}$, i.e., in the microseconds regime, interferes with echo formation. This reduction of the ${ }^{1} \mathrm{H}$ NMR SEI was exploited to investigate polymer dynamics in this intermediate motional regime. ${ }^{42}$ Detailed model calculations revealed that the temperature-dependent SEI has a minimum when the correlation time is similar to the echo delay, $\tau \approx \Delta_{\mathrm{e}}{ }^{43}$

In ${ }^{2} \mathrm{H}$ SLR and STE analyses, we probe the deuteron quadrupolar frequencies,

$$
\omega(\theta, \phi)= \pm \frac{\delta}{2}\left(3 \cos ^{2} \theta-1-\eta \sin ^{2} \theta \cos 2 \phi\right) .
$$

Here, the parameters $\delta$ and $\eta$ characterize the anisotropy and asymmetry of the quadrupolar interaction tensor of the deuteron, while the angles $\theta$ and $\phi$ describe the orientation of this tensor with respect to the applied magnetic field $B_{0}$. We expect that the deuterons of the studied $\varepsilon$-PLL- $\mathrm{D}_{2} \mathrm{O}$ mixture are distributed among the water $\mathrm{O}-\mathrm{D}$ and peptide N-D bonds because of chemical exchange. For these deuteron species, the interaction tensors are nearly axially symmetric $(\eta \approx 0)$, see below. Then, the quadrupolar frequencies are given by $^{25}$

$$
\omega\left(\theta_{n}\right) \approx \pm \frac{\delta_{n}}{2}\left(3 \cos ^{2} \theta_{n}-1\right) \propto P_{2}\left(\cos \theta_{n}\right),
$$

where the index $n$ is used to distinguish deuterons in various chemical environments and $\theta_{n}$ is the angle between the O-D or N-D bond and the $B_{0}$ field.

In ${ }^{2} \mathrm{H}$ SLR analysis, we probe the recovery of ${ }^{2} \mathrm{H}$ magnetization after either saturation or inversion. When dynamically distinguishable deuteron species $n$ coexist, ${ }^{2} \mathrm{H}$ magnetization may build up in separate steps. Considering also a possible nonexponentiality of the steps, ${ }^{44-46}$ we utilize a sum of stretched exponential or, equivalently, Kohlrausch-Williams-Watts (KWW) functions to analyze the observed buildup $M\left(t_{\mathrm{r}}\right)$. Explicitly, we fit saturation-recovery curves to

$$
M\left(t_{\mathrm{r}}\right)=M_{0}-\sum_{n} m_{n} \exp \left[-\left(\frac{t_{\mathrm{r}}}{T_{1, n}}\right)^{\beta_{1, n}}\right] .
$$

Here, $T_{1, n}$ and $\beta_{1, n}$ are the relaxation times and stretching parameters of the SLR steps, respectively. Moreover, the equilibrium magnetization is $M_{0}$, and the partial magnetizations of the deuteron species are denoted as $m_{n}$.

Quantitative analysis will be straightforward if a given SLR step is exponential $\left(\beta_{1, n}=1\right)$ and the related molecular reorientation is isotropic. Under such circumstances, the ${ }^{2} \mathrm{H}$ SLR time is given by ${ }^{38}$

$$
\frac{1}{T_{1, n}}=\frac{2}{15} \delta_{n}^{2}\left[J_{2, n}\left(\omega_{0}\right)+4 J_{2, n}\left(2 \omega_{0}\right)\right]
$$

Here, $J_{2, n}(\omega)$ is the spectral density that is related to the correlation function $F_{2, n}(t)$ of the O-D or N-D bonds by Fourier transformation. Furthermore, $\omega_{0}$ denotes the Larmor frequency. In temperature-dependent measurements, $T_{1, n}$ is minimum, when the correlation time of the probed molecular dynamics obeys $\omega_{0} \tau_{n}=0.6$ and, hence, amounts to $\sim 1 \mathrm{~ns}$ in our setup. In the temperature ranges below and above the minimum, shorter $T_{1, n}$ values are indicative of shorter and longer correlation times $\tau_{n}$, respectively. For a quantitative analysis, we exploit our knowledge from BDS and assume that $J_{2, n}(\omega)$ has a CC form, see Eq. (2), allowing us to determine the width parameter $\alpha_{n}$ from the minimum value of $T_{1, n}{ }^{27}$ Using the thus defined spectral density $J_{2, n}(\omega)$ in Eq. (6), we extract temperature-dependent correlation times $\tau_{n}$ from $T_{1, n}(T)$.

In ${ }^{2} \mathrm{H}$ STE studies, we directly correlate the quadrupolar frequencies $\omega$ and, thus, the bond orientations $\theta_{n}$ before and after a variable mixing time $t_{\mathrm{m}}$. Specifically, we measure the correlation function, ${ }^{25,27}$

$$
F_{2}^{\mathrm{ss}}\left(t_{\mathrm{m}}\right) \propto\left\langle\sin \left[\omega(t=0) t_{\mathrm{e}}\right] \sin \left[\omega\left(t=t_{\mathrm{m}}\right) t_{\mathrm{e}}\right]\right\rangle,
$$

which can be identified with $F_{2}\left(t_{\mathrm{m}}\right)$ in the limit of short evolution times $\omega t_{\mathrm{e}} \ll 1$; see Eq. (4). In general, the STE signal receives contributions from all deuterons in the studied sample. In our approach, we, however, use specifically chosen experimental parameters to ensure that the measured data are dominated by a particular deuteron species $n$, see below. Considering the fact that the STE amplitude decreases due to both molecular dynamics and spin relaxation, we fit the thus obtained and normalized STE decays to

$$
\left\{\left(1-F_{2}^{\infty}\right) \exp \left[-\left(t / \tau_{\mathrm{k}, n}\right)^{\beta_{\mathrm{k}, n}}\right]+F_{2}^{\infty}\right\} \exp \left(-t_{\mathrm{m}} / T_{1 \mathrm{Q}, n}\right) .
$$

Here, the first factor, a generalized KWW function, characterizes the decay due to reorientation dynamics, where the residual correlation $F_{2}^{\infty}$ is used to consider that the reorientation of deuteron species $n$ may be anisotropic so that it does not cause a complete loss of correlation or that other deuteron species may yield minor contributions, which do not decay due to rotational motion inside the experimental time window. The second factor describes the SLR damping of the STE amplitude, where $T_{1 \mathrm{Q}, n}$ is the SLR time of the alignment state of the spins, which exists during the mixing time of the performed STE experiments. ${ }^{27}$ For a determination of $T_{1 \mathrm{Q}, n}$, we exploit that SLR damping is independent of the evolution time and perform additional measurements for various $t_{\mathrm{e}}$ values (not shown) to obtain $T_{1 \mathrm{Q}, n}$ from global fits of the damping factors.

Striving for straightforward comparison of correlation times from multiple approaches, we need to consider that various experimental techniques probe different aspects of distributed dynamics. The abovedescribed CC analyses of BDS and SLR data yield peak correlation times $\tau_{n}$. Therefore, we calculate these time constants also from the KWW fits of the STE decays. Specifically, peak correlation times $\tau_{n}$ can be obtained from the fit parameters according to ${ }^{3}$

$$
\tau_{n}=\tau_{\mathrm{k}, n}\left(1.785-0.871 \beta_{\mathrm{k}, n}-0.029 \beta_{\mathrm{k}, n}^{2}+0.114 \beta_{\mathrm{k}, n}^{3}\right) .
$$

\section{EXPERIMENTAL DETAILS}

The studied aqueous solutions were prepared by mixing $\varepsilon$ PLL with $\mathrm{H}_{2} \mathrm{O}$ or $\mathrm{D}_{2} \mathrm{O}$. The peptide has a molecular weight of $M_{\mathrm{n}}=4090 \mathrm{~g} / \mathrm{mol}$ and consists of $\sim 32$ lysine monomers, $\left[\mathrm{CO}-\mathrm{CH}\left(\mathrm{NH}_{2}\right)-\left(\mathrm{CH}_{2}\right)_{4}-\mathrm{NH}\right]{ }^{47}$ It was kindly supplied by JNC Corporation (Japan). Before using, $\varepsilon$-PLL was purified utilizing an ion transfer resin (AG-501-X8, Bio-Rad laboratories). To avoid crystallization of excess water and to observe the vitrification of a fully amorphous system, ${ }^{22-24}$ we use moderate water concentrations of $c_{\mathrm{W}}$ $=33 \mathrm{wt} \%$ for $\varepsilon$-PLL- $\mathrm{H}_{2} \mathrm{O}$ and $c_{\mathrm{w}}=35 \mathrm{wt} \%$ for $\varepsilon$-PLL- $\mathrm{D}_{2} \mathrm{O}$. The solutions were sealed for 3 months to achieve a homogeneous water distribution. The $\mathrm{pH}$ value of the prepared solutions is 10 without addition of any buffer or salt. 

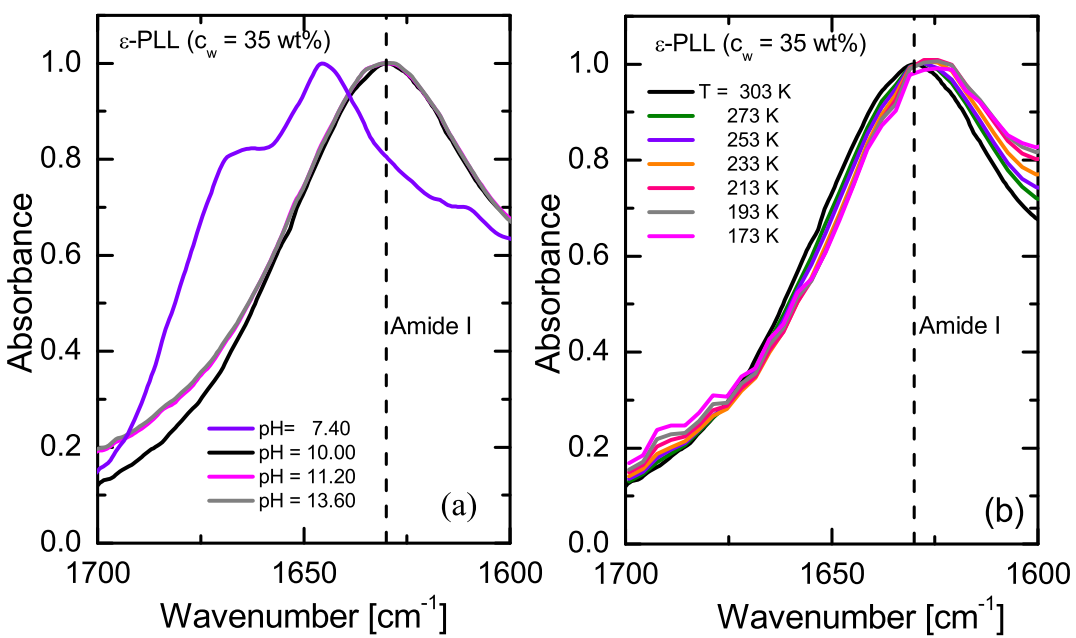

FIG. 1. Infrared spectra of $\varepsilon-P L L-\mathrm{H}_{2} \mathrm{O}$ (a) at different $\mathrm{pH}$ values and (b) at different temperatures for a sample, which, such as the BDS and NMR samples, has $\mathrm{pH}=10$ at room temperature. The dashed lines at $1630 \mathrm{~cm}^{-1}$ mark the amide I band position characteristic of the $\beta$-sheet structure. The conformation of the peptide changes with $\mathrm{pH}$, but it has $\beta$-sheet conformation in the studied BDS and NMR samples over the whole temperature range.
Figure 1 shows the amide I band of the infrared response, which is an indicator of the peptide conformation, for $\mathcal{E}$-PLL solutions at different $\mathrm{pH}$ values and temperatures. The amide I band found at $1630 \mathrm{~cm}^{-1}$ (see dashed lines) indicates a $\beta$-sheet conformation. ${ }^{23,48}$ The conformation of $\varepsilon$-PLL changes at $\mathrm{pH}=7$, where it is a mix of $\beta$-sheets and $\beta$-turns, but at $\mathrm{pH}=10$ or higher $\mathrm{pH}$ values, there is a $\beta$-sheet conformation even when decreasing the temperature. Thus, $\varepsilon$-PLL has this secondary structure in the present BDS and NMR studies.

Differential scanning calorimetry measurements, which were performed on a DSC Q-2000 from TA Instruments at cooling and heating rates of $10 \mathrm{~K} / \mathrm{min}$, yielded an onset for the glass transition temperature of $T_{\mathrm{g}}=222.8 \mathrm{~K}$ for $\varepsilon-\mathrm{PLL}-\mathrm{H}_{2} \mathrm{O}$, while crystallization did not occur. In BDS, we measured the complex dielectric permittivity, $\varepsilon^{*}(\omega)=\varepsilon^{\prime}(\omega)-i \varepsilon^{\prime \prime}(\omega)$ in a wide frequency range $\left(\sim 10^{-1}\right.$ to $\left.10^{10} \mathrm{~Hz}\right)$ by combining several dielectric techniques, as described in more detail in the previous work. ${ }^{22}$ The thickness of the samples was $0.1 \mathrm{~mm}$.

${ }^{1} \mathrm{H}$ NMR experiments were performed on a home-built spectrometer operating at a ${ }^{1} \mathrm{H}$ Larmor frequency of $\omega_{0}=2 \pi \cdot 91.2 \mathrm{MHz}$. ${ }^{1} \mathrm{H}$ NMR spectra were obtained from free induction decays. The mixed TREV4 pulse sequence, ${ }^{49} 90_{\mathrm{x}}^{\circ}-\Delta_{\mathrm{e}}-90_{\mathrm{y}}^{\circ}-\mathrm{CW}_{\mathrm{x}}\left(\Delta_{\mathrm{e}}\right)$ $-\mathrm{CW}_{-\mathrm{x}}\left(2 \Delta_{\mathrm{e}}\right)-90_{\mathrm{y}}^{\circ}-\Delta_{\mathrm{e}}$, was applied to determine temperaturedependent echo intensities for different echo delays $\Delta_{\mathrm{e}}$. The $90^{\circ}$ pulse length was set to $0.6 \mu \mathrm{s}$, and compared to this, a $-20 \mathrm{~dB}$ attenuation was used for the continuous wave $(\mathrm{CW})$ irradiation. The switching time between different phases and attenuations was $\sim 200$ ns.

${ }^{2} \mathrm{H}$ NMR measurements were performed using a homebuilt spectrometer working at a ${ }^{2} \mathrm{H}$ Larmor frequency of $\omega_{0}$ $=2 \pi \cdot 46.7 \mathrm{MHz}$. For ${ }^{2} \mathrm{H}$ SLR and LSA measurements, we used the saturation-recovery or inversion-recovery sequences together with a solid-echo readout sequence. In ${ }^{2} \mathrm{H}$ STE experiments, we employed a three-pulse sequence $90_{\mathrm{y}}^{\circ}-t_{\mathrm{e}}-45_{\mathrm{x}}^{\circ}-t_{\mathrm{m}}-45_{\mathrm{x}}^{\circ}-t_{\mathrm{e}}$, which divides the experimental time into two evolution times $t_{\mathrm{e}}$ sandwiching a longer variable mixing time $t_{\mathrm{m}}$. To refocus the STE signal outside the dead time of the receiver, we applied a fourth pulse. ${ }^{25,27}$ Further details of the used NMR setups and methods can be found in previous studies. $^{30,31,50}$

\section{RESULTS}

\section{A. Broadband dielectric spectroscopy studies}

BDS was proved a valuable tool to investigate aqueous $\varepsilon$-PLL solutions. Previous studies ${ }^{2-24}$ found that the dielectric spectra show four relaxation processes $\mathrm{P} 1-\mathrm{P} 4$ and determined the properties of these processes in a wide range of concentrations. The respective correlation times were obtained by fitting to a conductivity term and a superposition of CC functions; see Eq. (2). Here, we perform an analogous analysis for $\varepsilon-\mathrm{PLL}-\mathrm{H}_{2} \mathrm{O}(33 \mathrm{wt} \%)$. The obtained correlation times $\tau_{n}$ are shown in Fig. 2. The slowest process $\mathrm{P} 4$ shows the Vogel-Fulcher-Tammann temperature dependence and has a correlation time of $\sim 10^{2} \mathrm{~s}$ at $T_{\mathrm{g}}$. Based on similar observations, $\mathrm{P} 4$ was recently attributed to the structural $(\alpha)$ relaxation of the solute. ${ }^{22-24}$ Processes P3 and P2 are observed in both the liquid and glassy states, but their temperature dependencies change at $T_{\mathrm{g}}$. These behaviors are again consistent with those reported in previous studies. ${ }^{22-24}$ There, several observations, in particular

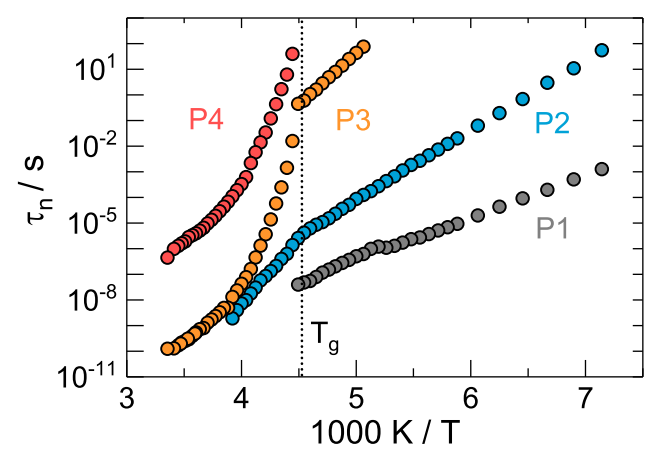

FIG. 2. Correlation times $\tau_{n}$ of $\varepsilon$-PLL- $\mathrm{H}_{2} \mathrm{O}$ from BDS. The dielectric loss spectra $\varepsilon^{\prime \prime}(v)$ show four relaxation processes denoted as P1-P4. The respective correlation times were obtained by fitting to Eq. (2), as described in detail in previous studies. ${ }^{22-24}$ The vertical dashed line indicates the glass transition temperature $T_{\mathrm{g}}=222.8 \mathrm{~K}$. 
concentration-dependent properties of the processes and comparisons with findings for other aqueous solutions, led to the conclusions that P3 is a coupled solvent-solute process and P2 is the Johari-Goldstein $\beta$ process of water in solutions. Finally, the fastest process P1 has Arrhenius temperature dependence. However, this relaxation is very weak and appears as a small shoulder rather than a separate peak in the dielectric loss $\varepsilon^{\prime \prime}(\omega)$, hampering detailed analysis and assignment.

\section{B. Nuclear magnetic resonance studies}

\section{Proton and deuteron species in the samples}

Prior to presenting ${ }^{1} \mathrm{H}$ and ${ }^{2} \mathrm{H}$ NMR results, it is useful to provide some basic information about the protons and deuterons in the studied $\varepsilon$-PLL- $\mathrm{H}_{2} \mathrm{O} / \mathrm{D}_{2} \mathrm{O}$ samples. $\varepsilon$-PLL comprises both acidic and basic groups, which can be ionized, depending on the $\mathrm{pH}$ value. In our case of $\mathrm{pH}=10$, there are carboxylate and ammonium groups at the chain ends, whereas the amide and amine groups along the backbone are largely neutral; see Fig. 3 . In ${ }^{1} \mathrm{H}$ NMR studies, we, thus, observe protons of $\mathrm{H}_{2} \mathrm{O}$ and protons in $\mathrm{C}-\mathrm{H}$ or $\mathrm{N}-\mathrm{H}$ bonds of the peptide, where the latter form amide $(\mathrm{NH})$, amine $\left(\mathrm{NH}_{2}\right)$, or ammonium $\left(\mathrm{NH}_{3}\right)$ groups. For the used $\varepsilon$ - $\mathrm{PLL}-\mathrm{H}_{2} \mathrm{O}$ mixture, there are $\sim 63 \%$ peptide protons and $\sim 37 \%$ water protons. In ${ }^{1} \mathrm{H}$ NMR studies on $\varepsilon$-PLL- $\mathrm{D}_{2} \mathrm{O}$, we need to consider that the proton-deuteron exchange occurs at $\mathrm{N}-\mathrm{H}$ bonds, but not at $\mathrm{C}-\mathrm{H}$ bonds so that $\sim 25 \%$ of all peptide protons are exchangeable. Assuming that the latter are statistically distributed between peptide and water, we thus expect that $\sim 75 \%$ of all protons of $\varepsilon$-PLL- $\mathrm{D}_{2} \mathrm{O}$ are in nonpolar $\mathrm{C}-\mathrm{H}$ bonds of the peptide backbone and do not participate in chemical exchange, while $\sim 8 \%$ in polar $\mathrm{N}-\mathrm{H}$ bonds of the peptide and $\sim 17 \%$ in $\mathrm{O}-\mathrm{H}$ bonds of water are exchangeable. Hence, ${ }^{1} \mathrm{H}$ NMR results for $\mathcal{\varepsilon}$-PLL- $\mathrm{D}_{2} \mathrm{O}$ are governed by dynamics of nonpolar peptide backbone regions; see Fig. 3 . In ${ }^{2} \mathrm{H}$ NMR approaches to $\varepsilon$ - PLL- $\mathrm{D}_{2} \mathrm{O}$, we deal with deuterons of $\mathrm{D}_{2} \mathrm{O}$ and, due to proton-deuteron exchange, in amide $(\mathrm{ND})$, amine $\left(\mathrm{ND}_{2}\right)$, and ammonium $\left(\mathrm{ND}_{3}\right)$ groups of the peptide. Assuming again a statistical proton/deuteron distribution, we expect $\sim 69 \%$ water deuterons and $\sim 31 \%$ peptide deuterons in the studied mixture.

For the interpretation of our NMR data, the time scale of the proton/deuteron exchange is very important. It strongly differs for various chemical groups and environments and changes by orders of magnitude when changing the $\mathrm{pH}$ value or temperature. ${ }^{51-53}$ Since chemical exchange is negligible for $\mathrm{C}-\mathrm{H}$ bonds of $\varepsilon$ - PLL, their contributions to ${ }^{1} \mathrm{H}$ NMR signals yield straightforward information about the dynamics of the peptide and, in particular, of the nonpolar $\left(\mathrm{CH}_{2}\right)_{4}$ backbone regions. All other protons and deuterons of the studied samples are exchangeable. For these species, the individual contributions can be resolved in ${ }^{1} \mathrm{H}$ and ${ }^{2} \mathrm{H}$ NMR when the exchange is slow on the experimental time scale, while fast swaps

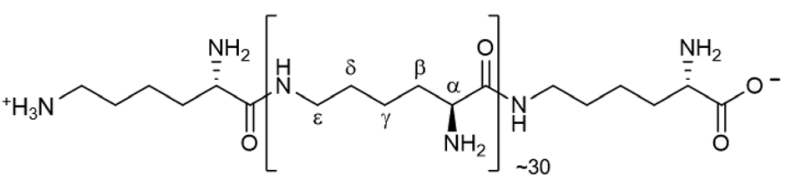

FIG. 3. Chemical structure of $\varepsilon-P L L$ in the zwitterionic form. We see that polar and nonpolar regions alternate along the peptide backbone. between molecular moieties result in common signals, which reflect average behaviors.

At neutral $\mathrm{pH}$ and ambient temperature, amide and ammonium hydrogens show exchange rates of $\sim 10^{2} \mathrm{~Hz}$ and $\sim 10^{4} \mathrm{~Hz}$, respectively. ${ }^{54,55}$ As the exchange is base catalyzed, it strongly speeds up when the $\mathrm{pH}$ value is increased. In our case of $\mathrm{pH}=10$, the rates amount to $\sim 10^{5} \mathrm{~Hz}$ for the amide groups and they are even higher for the amine and ammonium groups. ${ }^{56}$ Similar rates are expected for deuterons in such groups. Therefore, we envisage that the fastexchange limit applies and all exchangeable protons/deuterons contribute to common ${ }^{1} \mathrm{H} /{ }^{2} \mathrm{H}$ NMR signals at higher temperatures of the studied range. However, chemical exchange strongly slows down when the temperature is decreased, e.g., high activation energies of $\sim 70 \mathrm{~kJ} / \mathrm{mol}$ were found for amide protons. ${ }^{54,57}$ As a result, swaps between diverse species occur on time scales of seconds and longer at lower temperatures of the studied range so that the slowexchange limit applies and signals from various proton/deuteron species can be distinguished, which will allow us to resolve potentially diverse dynamical behaviors. In particular, it will be possible not only to ascertain differences between water and peptide reorientations but also to distinguish diverse behaviors of various peptide moieties. For example, we can exploit that amide groups solely probe the motion of the peptide backbone, while amine side groups and ammonium end groups also show rotations about their C$\mathrm{N}$ bond axes, which are fast and largely dominate their dynamical behaviors. $^{58,59}$

\section{2. ${ }^{2}$ H NMR studies}

First, we analyze the ${ }^{2} \mathrm{H}$ SLR of $\varepsilon$-PLL- $\mathrm{D}_{2} \mathrm{O}$ by performing saturation-recovery and inversion-recovery experiments. Figure 4 shows the buildup of the ${ }^{2} \mathrm{H}$ magnetization $M\left(t_{\mathrm{r}}\right)$ after saturation for various temperatures. The saturation-recovery curves involve several SLR steps at lower temperatures, while they are single exponential at $T>245 \mathrm{~K}$. These findings meet the expectations based on our previous knowledge about molecular dynamics and chemical exchange. At low temperatures, water and peptide show different dynamics and deuteron exchange is slow so that diverse deuteron species have different SLR behaviors. When increasing the temperature, the correlation times of different dynamical processes approach

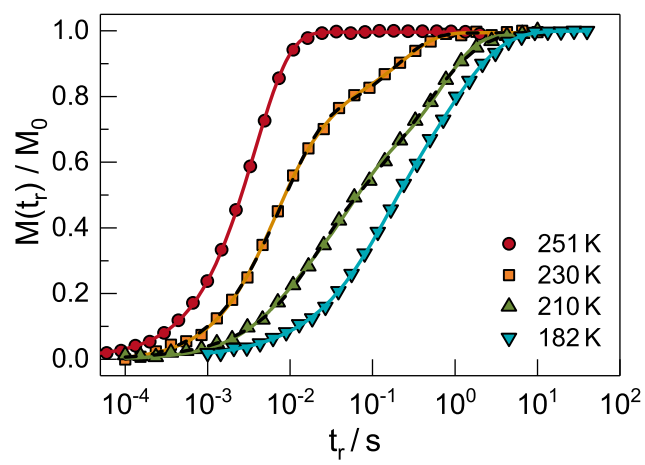

FIG. 4. Buildup of normalized ${ }^{2} \mathrm{H}$ magnetization $M\left(t_{r}\right) / M_{0}$ after saturation for $\varepsilon$ $\mathrm{PLL}-\mathrm{D}_{2} \mathrm{O}$ at various temperatures. The lines are fits to $\mathrm{Eq}$. (5). While a single SLR step was used to interpolate the data at $251 \mathrm{~K}$, three SLR steps were employed at the lower temperatures; see text for details. 
each other (see Fig. 2), and the chemical exchange speeds up, causing magnetization transfer between various deuteron species during the buildup process. As a consequence, all deuterons show a common SLR behavior at high temperatures. Considering these results, we fit $M\left(t_{\mathrm{r}}\right)$ to a sum of relaxation steps at lower temperatures [see Eq. (5)] and to a single exponential at $T>245 \mathrm{~K}$.

The ${ }^{2} \mathrm{H}$ SLR times $T_{1, n}$ obtained from these fits are presented in Fig. 5. The common high-temperature value $T_{1}$ shows a minimum near $260 \mathrm{~K}$. This finding indicates molecular dynamics on the time scale of the inverse Larmor frequency, $\tau \approx 1 \mathrm{~ns}$. However, this correlation time cannot be assigned to a specific deuteron species because the fast-exchange limit applies, and hence, an average relaxation behavior of all deuterons is observed in this temperature range. At $T<245 \mathrm{~K}$, different $T_{1, n}$ values can be distinguished indicative of the slow-exchange limit. This crossover from one-step to multistep ${ }^{2} \mathrm{H}$ SLR indicates that the time scale of the deuteron exchange crosses that of the magnetization buildup near $245 \mathrm{~K}$. However, the saturation-recovery results do not allow us to unambiguously determine the number and origin of the individual SLR steps. Therefore, we extract this information from inversion-recovery measurements before continuing the $T_{1, n}$ analysis.

In this approach, we combine inversion-recovery experiments with spectrally resolved analysis. Specifically, we first invert the equilibrium magnetization $M_{0}$ by applying a $180^{\circ}$ pulse, wait a variable relaxation delay $t_{\mathrm{r}}$ during which the magnetization may return to equilibrium, and, finally, record a ${ }^{2} \mathrm{H}$ NMR spectrum. Thus, the inverted and regular ${ }^{2} \mathrm{H}$ NMR line shapes are observed, when the recovery of the magnetization has not yet started for $t_{\mathrm{r}} \rightarrow 0$ and has been completed for $t_{\mathrm{r}} \rightarrow \infty$, respectively. At intermediate relaxation delays, deuteron species with $T_{1, n} \gg t_{\mathrm{r}}$ produce negative intensities, while those with $T_{1, n} \ll t_{\mathrm{r}}$ yield positive signals. Moreover, the contribution of a specific deuteron species is fully suppressed when the zero crossing of the corresponding magnetization occurs at the chosen relaxation delay $t_{\mathrm{r}}$.

Figure 6 shows ${ }^{2} \mathrm{H}$ NMR spectra obtained from such inversionrecovery experiments. Fully relaxed, i.e., regular ${ }^{2} \mathrm{H}$ NMR spectra

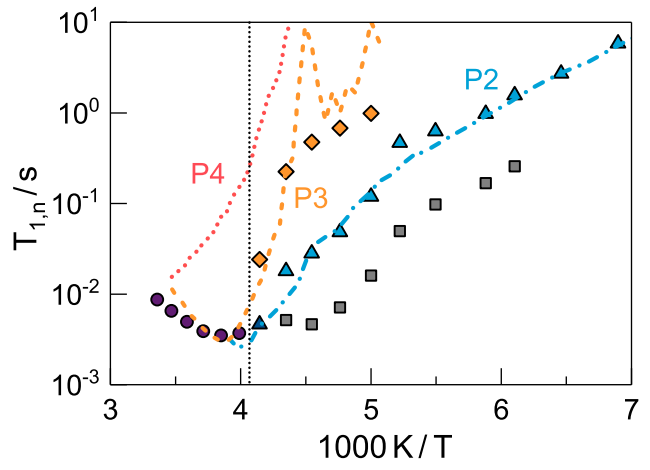

FIG. 5. ${ }^{2} \mathrm{H}$ SLR times $T_{1, n}$ of $\varepsilon-P L L-D_{2} \mathrm{O}$. The vertical dotted line separates temperature ranges above and below $\sim 245 \mathrm{~K}$ where SLR occurs in one and several steps, respectively. $T_{1, n}$ values calculated from our BDS results based on Eq. (6) are shown as lines. For the calculations, we used spectral densities $J_{2, n}(\omega)$ obtained from the CC parameterizations of the BDS processes and the Larmor frequency of $\omega_{0}=2 \pi \cdot 46 \mathrm{MHz}$

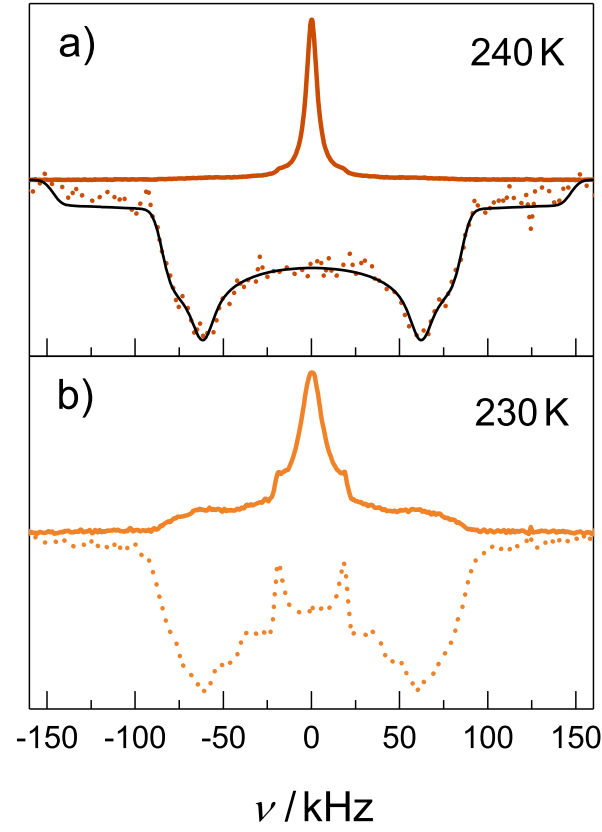

FIG. 6. Fully relaxed (solid lines) and partially relaxed (dotted lines) ${ }^{2} \mathrm{H}$ NMR solidecho spectra of $\varepsilon-P L L-D_{2} O$ at (a) $240 \mathrm{~K}$ and (b) $230 \mathrm{~K}$. In these measurements, we utilized a $180^{\circ}$ pulse to invert the magnetization and applied a solid-echo sequence after a relaxation delay $t_{\mathrm{r}}$ to record the spectrum. The fully relaxed spectra were obtained after full recovery of the equilibrium magnetization at $t_{\mathrm{r}} \gg T_{1, n}$. The partially relaxed spectra were measured for relaxation delays of $3.2 \mathrm{~ms}$ and $4.9 \mathrm{~ms}$ at $240 \mathrm{~K}$ and $230 \mathrm{~K}$, respectively. At these delays, the magnetization associated with the Lorentzian has its zero crossing, ensuring a suppression of this line-shape component. In panel (a), the black solid line is a fit of the partially relaxed spectrum to a Pake pattern, yielding $\delta=2 \pi \cdot 147 \mathrm{kHz}$ and $\eta=0.17$.

obtained at $t_{\mathrm{r}} \rightarrow \infty$ are compared with partially relaxed ones for shorter $t_{\mathrm{r}}$ values. In the latter measurements, we use relaxation delays at which the magnetization associated with the Lorentzian has its zero crossing so that this line-shape contribution is eliminated. The fully relaxed spectrum at $230 \mathrm{~K}$ comprises a Lorentzian line together with broad and narrow Pake spectra. The partially relaxed spectrum at this temperature shows the broad and narrow Pake spectra with negative and positive intensities, respectively, while the Lorentzian is suppressed, as expected. Thus, the magnetization associated with the broad Pake pattern relaxes slowly so that it is still in the inverted state, while that related to the narrow Pake pattern relaxes quickly and is already in the equilibrium state. Deuterons contributing to the Lorentzian line have a SLR time intermediate between that attributed to the two Pake spectra. Thus, each of the three line-shape components has a different $T_{1, n}$ value. At $240 \mathrm{~K}$, the regular spectrum is dominated by the Lorentzian line, while the narrow and broad Pake components are difficult to see. The partially relaxed spectrum features the negative broad Pake pattern of the slowly relaxing deuteron species, whereas the other two line-shape components are suppressed at the same time. These findings imply that the deuterons producing the Lorentzian line and the narrow Pake spectrum do no longer exhibit diverse $T_{1, n}$ values, but chemical exchange between these deuterons is sufficiently fast to establish a common SLR behavior at $240 \mathrm{~K}$, while deuterons associated with 
the broad Pake spectrum still show a different $T_{1, n}$ value. Exploiting this and other information from inversion-recovery experiments, we use a superposition of three SLR steps to interpolate the saturation-recovery curves $M\left(t_{\mathrm{r}}\right)$ in the temperature range 200$230 \mathrm{~K}$, while we perform monomodal or bimodal fits at higher or lower temperatures, see Fig. 4.

The combined information about the recovery rates and line shapes allows us to identify the various deuteron species. Two findings indicate that the broad Pake spectrum can be attributed to the ND groups. First, a fit of this line-shape component yields $\delta=2 \pi \cdot 147 \mathrm{kHz}$ and $\eta=0.17$, which are typical values of deuterons in amide bonds. ${ }^{60,61}$ Second, the finding that the deuteron species producing the broad Pake spectrum shows the longest $T_{1, n}$ value implies that it belongs to a slow molecular moiety, consistent with the fact that the amide groups probe the backbone dynamics of the peptide. The observation of a narrow Pake spectrum, on the other hand, is indicative of a deuteron species undergoing fast anisotropic motion. Therefore, we assign this line-shape component to the $\mathrm{ND}_{2} / \mathrm{ND}_{3}$ side/end groups, which show rapid rotation about the associated $\mathrm{C}-\mathrm{N}$ bonds. ${ }^{58,59}$ Finally, a Lorentzian line reveals fast isotropic motion, implying that this spectral pattern results from water deuterons.

Based on these assignments, we can estimate time constants $\tau_{\text {ex }}$ for the chemical exchange of different species from the respective onset temperatures of distinguishable ${ }^{2} \mathrm{H}$ SLR behaviors upon cooling. Revisiting Figs. 5 and 6, we see that the SLR of the ND groups is still the same as that of the other deuterons at $250 \mathrm{~K}$, but significantly different at $240 \mathrm{~K}$, indicating $\tau_{\mathrm{ex}} \approx T_{1} \approx 4 \mathrm{~ms}$ at $\sim 245 \mathrm{~K}$. Likewise, the SLR of the $\mathrm{ND}_{2} / \mathrm{ND}_{3}$ moieties separates from that of the water deuterons in the range $230-240 \mathrm{~K}$, implying that the exchange between these species occurs on a time scale $\tau_{\mathrm{ex}} \approx 4 \mathrm{~ms}$ at a somewhat lower temperature near $235 \mathrm{~K}$. This information about the time scale of chemical exchange will be useful in the following analyses of molecular dynamics.

The knowledge gained from the inversion-recovery experiments enables a more detailed discussion of the $T_{1, n}$ results in Fig. 5. At $200-230 \mathrm{~K}$, the longest and shortest $T_{1, n}$ values are associated with the $\mathrm{ND}$ and $\mathrm{ND}_{2} / \mathrm{ND}_{3}$ groups of the peptide, respectively, while the intermediate one is related to $\mathrm{D}_{2} \mathrm{O}$. For a quantitative analysis, we first focus on the exponential high-temperature relaxation, for which a $T_{1}$ minimum is observed. According to the BDS results, we use a CC spectral density and determine the width parameter $\alpha=0.5$ from the minimum height, indicating strong dynamical heterogeneity. Keeping this value fixed in the narrow temperature range of the present analysis, we obtain $\tau$ values from the $T_{1}$ data. In Fig. 7 , we see that the correlation times obtained from this SLR analysis agree with the common time constants of the BDS relaxation processes $\mathrm{P} 2$ and $\mathrm{P} 3$ in this temperature range. This is consistent with the expectation that the observed SLR behavior is common to water and peptide deuterons at high temperatures due to fast chemical exchange.

To rationalize the SLR behavior at lower temperatures, we take a different approach. Explicitly, we use the CC spectral densities from our BDS analysis in Eq. (6) to calculate expectations for the $T_{1, n}$ values. While doing so, we neglect differences between $F_{1}$ and $F_{2}$ correlation functions and assume isotropic motion. In Fig. 5, we see that the $T_{1, n}$ times measured for the water deuterons agree with that calculated based on the CC spectral densities of $\mathrm{P} 2$. This coincidence

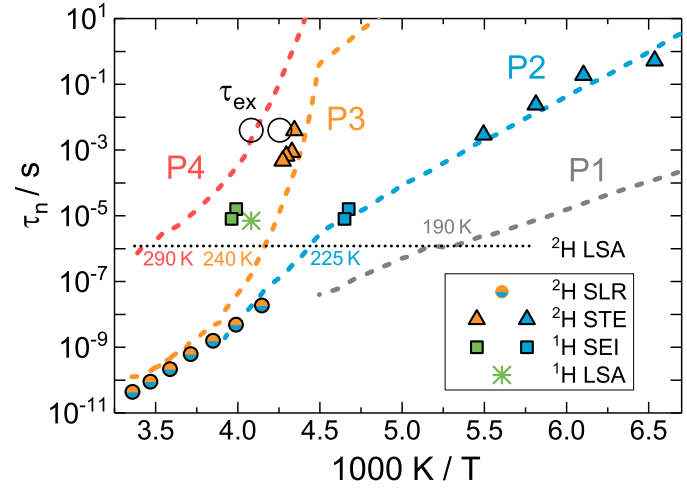

FIG. 7. Correlation times $\tau_{n}$ obtained from ${ }^{1} \mathrm{H}$ NMR and ${ }^{2} \mathrm{H}$ NMR studies on $\varepsilon$ $\mathrm{PLL}-\mathrm{H}_{2} \mathrm{O}$ and $\varepsilon-\mathrm{PLL}-\mathrm{D}_{2} \mathrm{O}$. For comparison, the correlation times from our BDS approach (see Fig. 2) are reproduced as dashed lines. The circles mark estimates for the time scale of the proton/deuteron exchange: left one, amide groups and right one, amine and ammonium groups. The horizontal dotted line indicates the time window of ${ }^{2} \mathrm{H}$ LSA. The BDS processes cross this time window at the indicated temperatures.

clearly confirms that $\mathrm{P} 2$ results from water reorientation. Likewise, a reasonable agreement is found between the observed $T_{1, n}$ values for the amide deuterons and the calculated ones for P3, implying that rotational motion of polar backbone groups is involved in this process. In this case, the weaker dielectric relaxation strength of P3 leads to larger uncertainties when determining the shape parameters of this process and, thus, to noticeable scattering of SLR behavior computed therefrom. By contrast, the $T_{1, n}$ times expected based on $\mathrm{P} 4$ are significantly longer than all measured values, and hence, this process is not observed in ${ }^{2} \mathrm{H}$ SLR studies of $\varepsilon$-PLL- $\mathrm{D}_{2} \mathrm{O}$. This finding suggests that $\mathrm{P} 4$ either does not involve polar peptide moieties or that chemical exchange at these sites hampers discrimination of their contributions from water contributions on the time scale of the magnetization buildup. In addition, one may wonder whether P1 is related to the rapid reorientation of $\mathrm{ND}_{2} / \mathrm{ND}_{3}$ groups. While the very weak dielectric relaxation strength and the clearly anisotropic reorientation mechanism hamper quantitative SLR analysis for these deuteron species, we will obtain information about this point from LSA approaches.

${ }^{2} \mathrm{H}$ LSA yields further information about the dynamical behavior of the peptide solution. Figure 8 shows ${ }^{2} \mathrm{H}$ NMR spectra of $\varepsilon$-PLL- $\mathrm{D}_{2} \mathrm{O}$, which were measured at various temperatures after full relaxation. We observe a broad solid-state Pake spectrum at $T \leq 140 \mathrm{~K}$ and a narrow liquid-like Lorentzian line at $T \geq 250 \mathrm{~K}$, while there is a superposition of broad and narrow line-shape components in the intermediate temperature range, reflecting the diverse dynamical behaviors of the different deuteron species.

Based on our SLR results, we expect that the low-temperature spectrum receives contributions from both water and peptide deuterons. The anisotropy and asymmetry parameters of the water deuterons, $\delta=2 \pi \cdot 161 \mathrm{kHz}$ and $\eta=0.1,{ }^{44,45}$ are, however, similar to the abovedetermined values for the peptide deuterons so that the respective line-shape components are not resolved. Thus, the Pake pattern at $T \leq 140 \mathrm{~K}$ indicates that both the $\mathrm{N}-\mathrm{D}$ and $\mathrm{O}-\mathrm{D}$ bonds are static on the time scale of the experiment, $\tau \approx 1 \mu \mathrm{s}$. When the temperature is increased, the narrow Pake spectrum gains intensity in the 


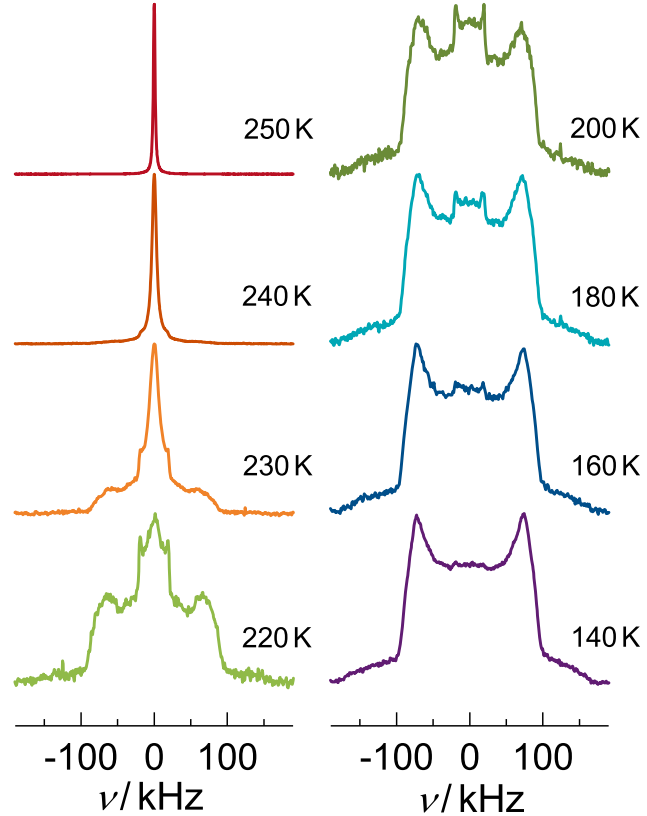

FIG. 8. ${ }^{2} \mathrm{H}$ NMR solid-echo spectra of $\varepsilon-P L L-\mathrm{D}_{2} \mathrm{O}$ at the indicated temperatures. The spectra were obtained from saturation-recovery experiments after complete relaxation of the magnetization.

range from $160 \mathrm{~K}$ to $220 \mathrm{~K}$, implying that the anisotropic reorientation of the amine and ammonium groups sets in on the time scale of the experiment. Hence, this motion is described by $\tau \approx 1 \mu \mathrm{s}$ at roughly $190 \mathrm{~K}$, but broadly distributed. At this temperature, the fastest BDS process crosses the time window of ${ }^{2} \mathrm{H}$ LSA, see Fig. 7, implying that $\mathrm{P} 1$ is related to this anisotropic side and end group motion.

Upon further heating, the broad and narrow Pake spectra are gradually replaced by the Lorentzian line from $\sim 220 \mathrm{~K}$ to $250 \mathrm{~K}$, implying that peptide and water deuterons are involved in reorientation dynamics, which become fast on the microseconds time scale. Comparison with the BDS correlation times in Fig. 7 indicates that $\mathrm{P} 4$ is too slow to produce the observed line narrowing, while $\mathrm{P} 2$ and $\mathrm{P} 3$ cross the time scale of ${ }^{2} \mathrm{H}$ LSA at $225 \mathrm{~K}$ and $240 \mathrm{~K}$, respectively. Hence, water and peptide dynamics involved in the latter processes cause the Pake-Lorentzian crossover. In particular, the absence of any broad line-shape component at $T \geq 250 \mathrm{~K}$ indicates that all deuterons of $\varepsilon$-PLL- $\mathrm{D}_{2} \mathrm{O}$ are involved in dynamical processes other than that associated with $\mathrm{P} 4$. We note that additional line narrowing due to deuteron exchange between peptide and water may not be fully excluded. However, we expect that the effects on the observed line-shape transition are weak because our ${ }^{2} \mathrm{H}$ SLR analysis indicated that the deuteron exchange is slow on the microseconds time scale of ${ }^{2} \mathrm{H}$ LSA at $220-250 \mathrm{~K}$; see Fig. 7.

${ }^{2} \mathrm{H}$ STE experiments provide access to correlation functions of slow molecular reorientation. In Fig. 9, we display $F_{2}^{s \mathrm{~s}}\left(t_{\mathrm{m}}\right)$ measured for $\varepsilon$-PLL- $\mathrm{D}_{2} \mathrm{O}$ at low temperatures after full relaxation. We observe strongly stretched decays, which shift to longer times upon cooling. Considering that ${ }^{2} \mathrm{H}$ STE signals decay due to molecular dynamics and spin relaxation, we fit the decays to a modified KWW function multiplied by SLR damping, see Eq. (8). From the fit results,

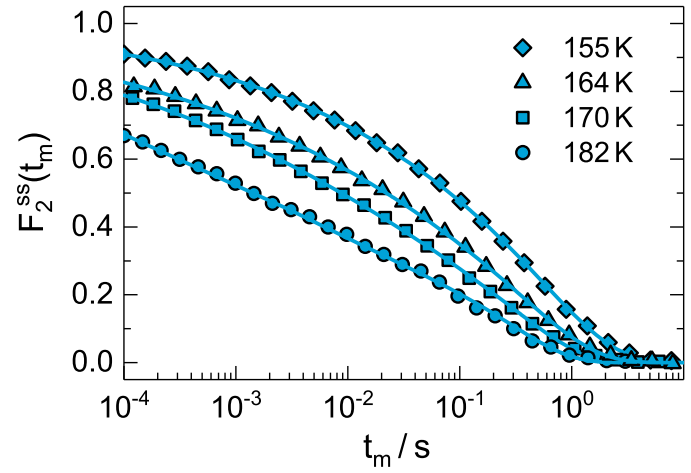

FIG. 9. Temperature-dependent rotational correlation functions $F_{2}^{\text {ss }}\left(t_{\mathrm{m}}\right)$ of $\varepsilon$-PLL$\mathrm{D}_{2} \mathrm{O}$ from ${ }^{2} \mathrm{H}$ STE experiments after full relaxation of the magnetization. The evolution time was set to $t_{\mathrm{e}}=5 \mu \mathrm{s}$ so that $F_{2}^{\mathrm{ss}}\left(t_{\mathrm{m}}\right) \approx F_{2}\left(t_{\mathrm{m}}\right)$. The lines are fits to a generalized KWW function multiplied by SLR damping, see Eq. (8).

we calculate peak correlation times $\tau$ according to Eq. (9). In Fig. 7, it can be seen that the NMR results agree with the BDS relaxation times for P2. As the ${ }^{2} \mathrm{H}$ STE signal is dominated by the $\mathrm{D}_{2} \mathrm{O}$ contribution, this agreement further confirms that water reorientation is at the origin of this BDS process. Based on the observation that the orientational correlation is lost to a large extent in the accessible dynamic range, we can, moreover, infer that the reorientation process is quasi-isotropic and involves most water molecules. In other words, even well below $T_{\mathrm{g}}$, there is, if at all, only a small fraction of water molecules, which show appreciably anisotropic reorientation and/or are essentially immobile. Hence, our NMR results reveal that P2 is caused by an essentially isotropic water reorientation, which involves the vast majority of water molecules and occurs decoupled from the $\alpha$ relaxation of the peptide. Nevertheless, the water molecules show prominent dynamical heterogeneity, as indicated by the very stretched correlation functions, and strongly enhance the mobility of the peptide. ${ }^{22-24}$ Thus, they behave as plasticizer molecules, which strongly reduce the glass transition temperatures $T_{\mathrm{g}}$ of polymers and show heterogeneous isotropic reorientation in vitrified polymer matrices. $^{62-64}$

In Fig. 10, we present $F_{2}^{\text {ss }}\left(t_{\mathrm{m}}\right)$ obtained from ${ }^{2} \mathrm{H}$ STE studies on $\varepsilon$-PLL- $\mathrm{D}_{2} \mathrm{O}$ at higher temperatures of $\sim 233 \mathrm{~K}$. In these measurements, we use a short relaxation delay $t_{\mathrm{r}}$ between a saturation of the magnetization and the acquisition of the signal to single out the contribution of the $\mathrm{ND}_{2} / \mathrm{ND}_{3}$ groups with a short $T_{1, n}$ time. To validate this approach, the Fourier transform of a STE time signal is shown in the inset. We observe the narrow Pake spectrum of the amine and ammonium deuterons, confirming successful selection of these species. $F_{2}^{s s}\left(t_{\mathrm{m}}\right)$ decreases on a time scale $t_{\mathrm{m}} \approx 1 \mathrm{~ms}$, which is mildly shorter than that of spin relaxation and chemical exchange, the latter occurring at $\tau_{\mathrm{ex}} \approx 4 \mathrm{~ms}$ at a slightly higher temperature of $\sim 235 \mathrm{~K}$, as discussed above. Therefore, we conclude that the STE decays from these partially relaxed measurements, at least, their initial parts, yield insights into peptide dynamics, although other damping mechanisms cannot be excluded.

For further interpretation, we need to consider the fast reorientation of the $\mathrm{ND}_{2} / \mathrm{ND}_{3}$ groups about the associated $\mathrm{C}-\mathrm{N}$ bonds at the studied temperatures; see Fig. 8. As a consequence, the 


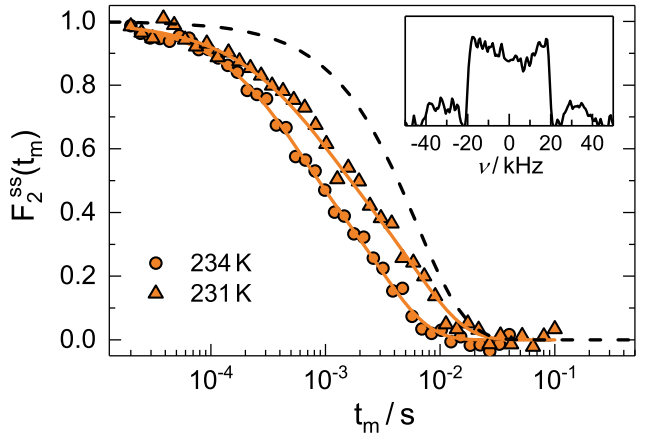

FIG. 10. Temperature-dependent rotational correlation functions $F_{2}^{s s}\left(t_{m}\right)$ of $\varepsilon$ PLL- $\mathrm{D}_{2} \mathrm{O}$ from ${ }^{2} \mathrm{H}$ STE experiments after partial relaxation of the magnetization. An evolution time of $t_{\mathrm{e}}=5 \mu \mathrm{s}$ was used so that $F_{2}^{\mathrm{ss}}\left(t_{\mathrm{m}}\right) \approx F_{2}\left(t_{\mathrm{m}}\right)$. Short relaxation delays $t_{r}$ were used to ensure that amine and ammonium deuterons with short $T_{1, n}$ times dominate the experimental result. The solid lines are fits to Eq. (8), and the dashed line indicates the SLR function at $231 \mathrm{~K}$. The inset shows the spectrum obtained from Fourier transformation of the STE signal for the shortest mixing time $t_{\mathrm{m}}$. It shows a narrow Pake spectrum, confirming successful selection of the $\mathrm{ND}_{2} / \mathrm{ND}_{3}$ group signal.

quadrupolar interaction tensor is motionally averaged and has its $z$ principal axis parallel to the $\mathrm{C}-\mathrm{N}$ bond axis. Under these circumstances, $F_{2}^{\mathrm{ss}}\left(t_{\mathrm{m}}\right)$ does not probe the reorientation of the N-D bonds but rather that of the $\mathrm{C}-\mathrm{N}$ bonds, which, in turn, reflects dynamics of polar backbone groups. Altogether, the STE decays suggest that polar groups in the $\mathcal{E}$-PLL backbone show reorientation dynamics in the milliseconds regime at the studied temperatures. The peak correlation times obtained from KWW fits are included in Fig. 7. We observe that the values are similar to the relaxation times of P3, implying that this BDS process involves a reorientation of the polar peptide segments. The obtained residual correlations $F_{2}^{\infty} \approx 0.5$ may suggest some anisotropy of the motion [see Eq. (8)], but this finding should be taken with care due to the interference of spin relaxation and chemical exchange at long mixing times $t_{\mathrm{m}}$.

\section{3. ${ }^{7}$ H NMR studies}

We move on to ${ }^{1} \mathrm{H}$ NMR studies. In Fig. 11, we see that the ${ }^{1} \mathrm{H}$ NMR spectra of $\varepsilon$-PLL- $\mathrm{D}_{2} \mathrm{O}$ comprise a broader and a narrower line over a wide temperature range, indicating proton species with different dynamics. When the temperature is decreased, the broader spectral component starts to split off from the narrower one near $260 \mathrm{~K}$. While the former rapidly widens down to $230 \mathrm{~K}$, where it reaches its static limit, the latter weakly broadens, but is expected do so at lower temperatures. The ${ }^{1} \mathrm{H}$ NMR spectra of $\varepsilon-\mathrm{PLL}-\mathrm{H}_{2} \mathrm{O}$ behave alike. The broader and narrower spectral components can be attributed to protons in less mobile peptide molecules and more mobile water molecules, respectively. In principle, the line shape may be somewhat affected by rapid chemical exchange between peptide and water. However, recalling that $\sim 75 \%$ of the ${ }^{1} \mathrm{H}$ NMR signals of $\varepsilon$-PLL- $\mathrm{D}_{2} \mathrm{O}$ result from nonexchangeable protons in $\mathrm{C}-\mathrm{H}$ bonds, we expect that the observed motional narrowing of the broad spectral component is dominated by reorientation dynamics of nonpolar regions of the $\varepsilon$-PLL backbone.

For quantitative analysis, we fit the ${ }^{1} \mathrm{H}$ NMR spectra to a weighted superposition of two components. The narrower line can

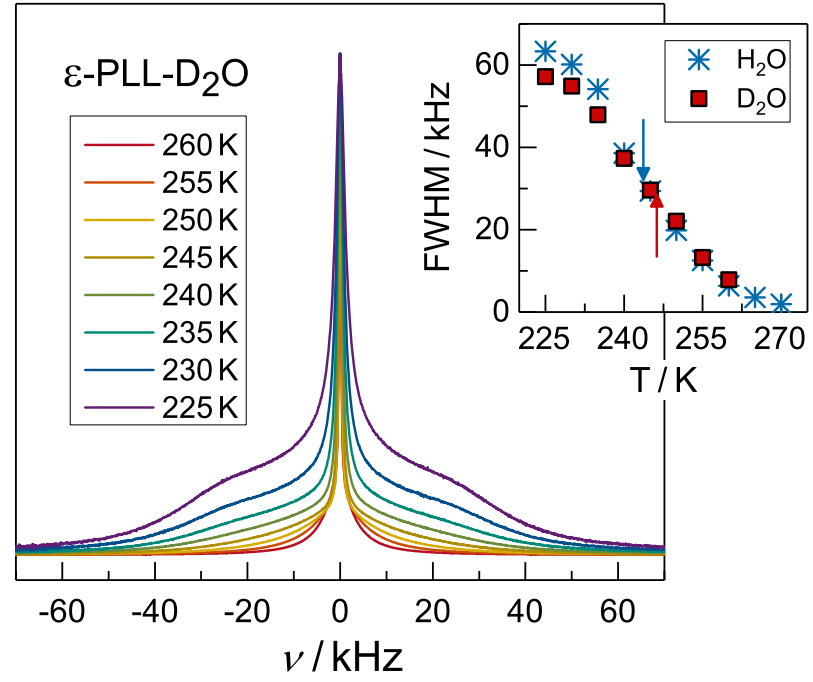

FIG. 11. ${ }^{1} \mathrm{H}$ NMR spectra of $\varepsilon-P L L-D_{2} \mathrm{O}$ at various temperatures. The inset shows the temperature-dependent full width at half maximum of the broad lineshape component, as obtained from fits of these results for $\varepsilon-P L L-D_{2} O$ and from analogous analysis for $\varepsilon$-PLL- $\mathrm{H}_{2} \mathrm{O}$.

be described by a Lorentzian function at all studied temperatures, while the broader line is interpolated with a pseudo-Voigt function, which allows us to consider that its shape evolves from Lorentzian to Gaussian when the temperature is decreased. In the inset of Fig. 11, the full width at half maximum $\Delta v$ of the broader spectral component is plotted as a function of temperature. Evidently, motional narrowing of the broad line is similar for $\varepsilon$-PLL- $\mathrm{D}_{2} \mathrm{O}$ and $\varepsilon-\mathrm{PLL}-\mathrm{H}_{2} \mathrm{O}$. Below $225 \mathrm{~K}$, a line width of $\Delta v_{0} \approx 60 \mathrm{kHz}$ is found. In this temperature range, all peptide protons are immobile on the microseconds time scale of the line-shape experiment. When the temperature is increased, the line width rapidly narrows. Above $260 \mathrm{~K}$, the absence of a broad spectral component reveals that there are no protons left that are immobile on the experimental time scale. Thus, the peptide backbone shows quasi-isotropic and sub-microseconds dynamics at these temperatures. We reiterate that the major contribution to the ${ }^{1} \mathrm{H}$ NMR signal stems from nonexchangeable protons in nonpolar methylene groups, and hence, this conclusion is not impaired by proton exchange.

To determine correlation times from ${ }^{1} \mathrm{H}$ motional narrowing, it proved useful to determine at which temperature $T_{\frac{1}{2}}$ the line width $\Delta v$ is reduced to $\frac{1}{2} \Delta v_{0}$ and to exploit that, at this temperature, the correlation time obeys $\Delta v_{0} \tau=c$, where the value of $c$ was argued to be 0.23 or $0.64 .^{38,39,65}$ We find $T_{\frac{1}{2}} \approx 245 \mathrm{~K}$ for $\varepsilon$-PLL- $\mathrm{D}_{2} \mathrm{O}$ and $\varepsilon$-PLL- $\mathrm{H}_{2} \mathrm{O}$, implying a correlation time of $\tau \approx 7 \mu$ for $\Delta v_{0}=60 \mathrm{kHz}$ and an average value of $c=0.44$. However, this value should be considered as a rough estimate because the used relation was derived for an exponential correlation function, and deviations are expected in our case of significant nonexponentiality. ${ }^{66}$

Alternatively, we can obtain ${ }^{1} \mathrm{H}$ NMR correlation times when we exploit that the efficiency of spin echo pulse sequences is strongly reduced by molecular dynamics in the intermediate motional regime and determine the temperature-dependent echo amplitude. Figure 12 shows ${ }^{1} \mathrm{H}$ SEI data of $\varepsilon$-PLL- $\mathrm{H}_{2} \mathrm{O}$ and $\varepsilon$-PLL- $\mathrm{D}_{2} \mathrm{O}$, which 


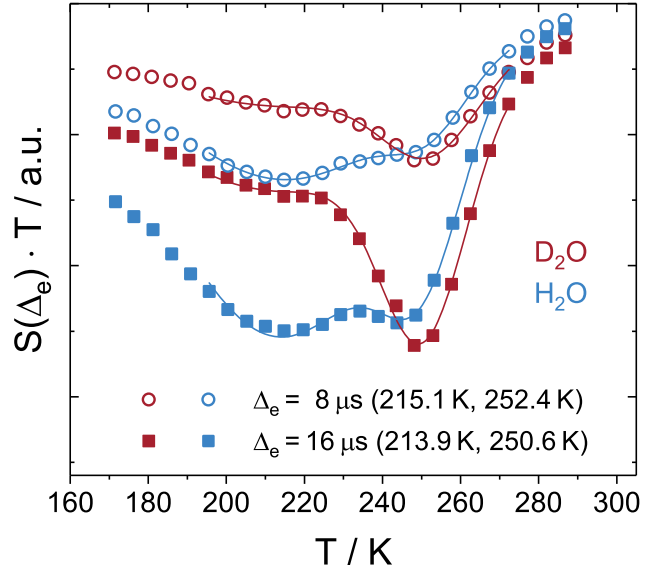

FIG. 12. Temperature-dependent ${ }^{1} \mathrm{H}$ SEI data of $\varepsilon$-PLL- $\mathrm{H}_{2} \mathrm{O}$ (blue) and $\varepsilon$-PLL$\mathrm{D}_{2} \mathrm{O}$ (red) for two echo delays, $\Delta_{\mathrm{e}}=8 \mu \mathrm{s}$ and $\Delta_{\mathrm{e}}=16 \mu \mathrm{s}$, in the used mixed TREV4 pulse sequence. The measured echo amplitudes $S\left(\Delta_{\mathrm{e}}\right)$ are multiplied by the respective temperature $T$ to correct for the Curie factor. The solid lines are global fits to a superposition of two Gaussian functions; see the text for details. The indicated temperatures characterize the obtained positions of the minima.

were corrected for the Curie factor. We observe that, as a result of spin-spin relaxation, the echo amplitude is generally lower for a longer echo delay $\Delta_{\mathrm{e}}$. More interestingly, for both studied samples and echo delays, the SEI shows two minima, a broader one near $215 \mathrm{~K}$ and a narrower one near $250 \mathrm{~K}$. While the intensities at both minima are similar for $\varepsilon-\mathrm{PLL}-\mathrm{H}_{2} \mathrm{O}$, the minimum at the higher temperature is much deeper than that at the lower temperature for $\varepsilon$ PLL- $\mathrm{D}_{2} \mathrm{O}$. Considering the different fractions of water and peptide protons in the samples, these findings indicate that the SEI minimum at $\sim 215 \mathrm{~K}$ results from water dynamics, whereas that at $\sim 250 \mathrm{~K}$ is largely due to dynamics of the peptide backbone, in particular, of its nonpolar regions. Comparing the results for both echo delays in more detail, we find that the SEI minima are located at slightly lower temperatures for larger $\Delta_{\mathrm{e}}$ values, consistent with the fact that they occur at $\tau \approx \Delta_{\mathrm{e}}{ }^{42,43}$

For a more precise determination of the positions of the minima, we interpolate the SEI data in an appropriate temperature range with a weighted superposition of two Gaussian functions. Specifically, for each $\Delta_{\mathrm{e}}$ value, we simultaneously fit the results for $\varepsilon$-PLL$\mathrm{H}_{2} \mathrm{O}$ and $\varepsilon$-PLL- $\mathrm{D}_{2} \mathrm{O}$ treating the minimum positions as shared parameters. This approach consistently describes the SEI data for both samples, see Fig. 12. The resulting correlation times $\tau=\Delta_{\mathrm{e}}$ are included in Fig. 7. We find that the ${ }^{1} \mathrm{H}$ SEI correlation times of water dynamics well interpolate between the ${ }^{2} \mathrm{H}$ SLR and STE results at higher and lower temperatures, respectively, and agree with the BDS relaxation times of $\mathrm{P} 2$. These findings confirm the validity of the present ${ }^{1} \mathrm{H}$ SEI approach and show once more that water reorientation is at the origin of $\mathrm{P} 2$. The ${ }^{1} \mathrm{H}$ SEI results for peptide dynamics are roughly consistent with the estimate from ${ }^{1} \mathrm{H}$ LSA. However, the ${ }^{1} \mathrm{H}$ NMR correlation times agree with none of the BDS relaxation times, but they are located between that of processes $\mathrm{P} 3$ and $\mathrm{P} 4$. On the first glance, this finding is puzzling because chemical exchange, which can, in principle, average between $\mathrm{P} 3$ and $\mathrm{P} 4$ behaviors, should be of limited relevance, in particular, in ${ }^{1} \mathrm{H}$ NMR studies on $\varepsilon$-PLL- $\mathrm{D}_{2} \mathrm{O}$, where $\sim 75 \%$ of the signals result from nonexchangeable peptide protons. One should, however, consider that BDS, such as the abovementioned ${ }^{2} \mathrm{H}$ NMR studies, probe the reorientation of polar peptide groups, while the ${ }^{1} \mathrm{H}$ LSA and SEI data are governed by that of the nonpolar backbone moieties. Due to very different interactions of nonpolar and polar peptide parts with water, diverse dynamical behaviors of these entities are actually expected rather than unexpected.

\section{SUMMARY}

We combined BDS and NMR approaches to ascertain molecular reorientations in aqueous $\varepsilon$-PLL solutions. In agreement with findings in previous studies, ${ }^{22-24}$ the dielectric spectra feature four relaxation processes, $\mathrm{P} 1-\mathrm{P} 4$, which are related to molecular dynamics. The fastest process P1 obeys the Arrhenius law, while the slowest process $\mathrm{P} 4$ shows prominent non-Arrhenius behavior. For the intermediate processes $\mathrm{P} 2$ and $\mathrm{P} 3$, the temperature dependence strongly changes at $T_{\mathrm{g}}=222.8 \mathrm{~K}$, in analogy to the findings for secondary relaxations in various glass-forming mixtures. ${ }^{5,11,20,23,67}$

Likewise, ${ }^{1} \mathrm{H}$ and ${ }^{2} \mathrm{H}$ NMR studies on $\varepsilon$-PLL- $\mathrm{H}_{2} \mathrm{O}$ and $\varepsilon$ - PLL- $\mathrm{D}_{2} \mathrm{O}$ mixtures revealed a very complex relaxation scenario. The NMR scenario is even more complex than that in analogous approaches to hydrated proteins ${ }^{29-32}$ because the amino acid lysine features $\mathrm{ND}_{2}$ side groups, which significantly contribute to the NMR signals in our measurements, and show rotational dynamics, which differs from backbone and water motions. Therefore, it was important to exploit that protons and deuterons in various molecular moieties can be distinguished based on different magnetization buildups and line shapes. However, fast proton/deuteron exchange between peptide and water still complicates the analysis of the experimental data.

Nonetheless, based on short ${ }^{2} \mathrm{H} T_{1, n}$ times and narrow ${ }^{2} \mathrm{H}$ Pake spectra, it became clear that the amine side groups and ammonium end groups of the peptide show rapid reorientation about their $\mathrm{C}-\mathrm{N}$ bond axes, which are most probably linked to BDS process $\mathrm{P} 1$. Moreover, various ${ }^{1} \mathrm{H}$ and ${ }^{2} \mathrm{H}$ NMR results indicated consistently that BDS process $\mathrm{P} 2$ results from water dynamics, in harmony with the assignment in previous studies. ${ }^{22-24}$ Particularly, ${ }^{2} \mathrm{H}$ LSA and STE analyses showed that the underlying reorientation is quasi-isotropic. Furthermore, these studies revealed that essentially all water molecules participate in water reorientation related to process $\mathrm{P} 2$ and retain high mobility even well below $T_{\mathrm{g}}$, although they show broad distributions of correlation times, as indicated by small CC width and KWW stretching parameters. Altogether, BDS process $\mathrm{P} 2$ can be assigned to a quasi-isotropic reorientation of large parts of the water fraction, which decouples from the $\alpha$ relaxation of the peptide fraction upon cooling but still enhances peptide mobility, resembling the dynamical behavior of the faster component of other dynamically highly asymmetric glass-forming mixtures.

Relating to process P3, our ${ }^{2} \mathrm{H}$ SLR, LSA, and STE studies unraveled that dynamics of polar peptide groups are involved, while they did not probe contributions from water dynamics. In other words, our ${ }^{2} \mathrm{H}$ NMR approaches did not yield evidence for a coexistence of peptide-decoupled and peptide-coupled water species, which show fast and slow reorientation dynamics and add to BDS processes $\mathrm{P} 2$ and $\mathrm{P} 3$, respectively. However, small water fractions 
$(<10 \%)$, which do not contribute to $\mathrm{P} 2$ but rather to $\mathrm{P} 3$, may remain undetected. Also, contributions from collective motions of water dipoles related to process $\mathrm{P} 3$ may be enhanced in BDS, e.g., via nonvanishing cross-term contributions, but missed in ${ }^{2} \mathrm{H}$ NMR, which probes single-particle correlation functions. Anyway, we expect that the dynamics of the polar peptide groups are strongly coupled to the motion of neighboring water molecules. BDS process $\mathrm{P} 4$ is not directly observed in the present NMR studies.

${ }^{1} \mathrm{H}$ LSA and SEI analyses provided access to the reorientation dynamics of the nonpolar regions in the peptide backbone. The correlation times of this motion are located between that of the BDS processes $\mathrm{P} 3$ and $\mathrm{P} 4$. We expect that this reorientation dynamics is not probed by BDS because it involves the $\left(\mathrm{CH}_{2}\right)_{4}$ segments, which do not carry dipole moments. Moreover, we argue that the dynamics of these nonpolar moieties differs from that of the polar ones because of diverse water couplings of the respective peptide parts. Hence, like proteins, $\varepsilon$-PLL shows diverse modes of motion, resulting in a complex and heterogeneous relaxation scenario. Before conclusions about the microscopic origins of processes $\mathrm{P} 3$ and $\mathrm{P} 4$ can be drawn, it is, however, necessary to further explore the role of chemical exchange on the observed BDS and NMR relaxation scenarios in future work. Notwithstanding, we expect that many aspects of the dynamical behavior of the studied peptide solution apply to other dynamically highly asymmetric mixtures, e.g., protein solutions or polymer-plasticizer systems.

\section{ACKNOWLEDGMENTS}

Financial support through the Grant Nos. Vo-905/8-2, PID2019-104650GB-C21, and LINKB20012 is gratefully acknowledged.

\section{DATA AVAILABILITY}

The data that support the findings of this study are available from the corresponding author upon reasonable request.

\section{REFERENCES}

${ }^{1}$ B. Bagchi, Chem. Rev. 105, 3197 (2005).

${ }^{2}$ P. Ball, Chem. Rev. 108, 74 (2008).

${ }^{3}$ C. A. Angell, Chem. Rev. 102, 2627 (2002).

${ }^{4}$ H. E. Stanley, S. V. Buldyrev, G. Franzese, P. Kumar, F. Mallamace, M. G. Mazza, K. Stokely, and L. Xu, J. Phys.: Condens. Matter 22, 284101 (2010).

${ }^{5}$ S. Capaccioli, K. L. Ngai, S. Ancherbak, P. A. Rolla, and N. Shinyashiki, J. Non-Cryst. Solids 357, 641 (2011).

${ }^{6}$ S. Cerveny, F. Mallamace, J. Swenson, M. Vogel, and L. Xu, Chem. Rev. 116, 7608 (2016).

${ }^{7}$ P. W. Fenimore, H. Frauenfelder, B. H. McMahon, and R. D. Young, Proc. Natl. Acad. Sci. U. S. A. 101, 14408 (2004)

${ }^{8}$ Y. Levy and J. N. Onuchic, Annu. Rev. Biophys. Biomol. Struct. 35, 389 (2006).

${ }^{9}$ M.-C. Bellissent-Funel, A. Hassanali, M. Havenith, R. Henchman, P. Pohl, F. Sterpone, D. van der Spoel, Y. Xu, and A. E. Garcia, Chem. Rev. 116, 7673 (2016).

${ }^{10}$ S. Khodadadi and A. P. Sokolov, Biochim. Biophys. Acta, Gen. Subj. 1861, 3546 (2017).

${ }^{11}$ J. Swenson and S. Cerveny, J. Phys.: Condens. Matter 27, 033102 (2015).

${ }^{12}$ M. Rosenstihl, K. Kämpf, F. Klameth, M. Sattig, and M. Vogel, J. Non-Cryst. Solids 407, 449 (2015)

${ }^{13}$ W. Doster, Eur. Phys. J. 37, 591 (2008).
${ }^{14}$ S. Khodadadi, J. H. Roh, A. Kisliuk, E. Mamontov, M. Tyagi, S. A. Woodson, R. M. Briber, and A. P. Sokolov, Biophys. J. 98, 1321 (2010).

${ }^{15}$ K. L. Ngai, S. Capaccioli, and A. Paciaroni, Biochim. Biophys. Acta, Gen. Subj. 1861, 3553 (2017).

${ }^{16}$ J. Swenson, H. Jansson, and R. Bergman, Phys. Rev. Lett. 96, 247802 (2006).

${ }^{17}$ S. Khodadadi, S. Pawlus, and A. P. Sokolov, J. Phys. Chem. B 112, 14273 (2008).

${ }^{18}$ M. Vogel, J. Phys. Chem. B 113, 9386 (2009).

${ }^{19}$ M. G. Mazza, K. Stokely, S. E. Pagnotta, F. Bruni, H. E. Stanley, and G. Franzese, Proc. Natl. Acad. Sci. U. S. A. 108, 19873 (2011).

${ }^{20}$ S. Cerveny, A. Alegria, and J. Colmenero, Phys. Rev. B 77, 031803 (2008).

${ }^{21}$ K. L. Ngai, S. Capaccioli, and A. Paciaroni, Chem. Phys. 424, 37 (2013).

${ }^{22}$ I. Combarro-Placios, C. Olsson, C. S. Kamma-Lorger, J. Swenson, and S. Cerveny, J. Chem. Phys. 150, 124902 (2019).

${ }^{23}$ S. Cerveny and J. Swenson, J. Chem. Phys. 150, 234904 (2019).

${ }^{24}$ S. Cerveny, I. Combarro-Palacios, and J. Swenson, J. Phys. Chem. Lett. 7, 4093 (2016).

${ }^{25}$ K. Schmidt-Rohr and H. W. Spiess, Multidimensional Solid-State NMR and Polymers (Academic Press, 1994).

${ }^{26}$ R. Kimmich, NMR-Tomography, Diffusometry, Relaxometry (Springer, Berlin, 1997).

${ }^{27}$ R. Böhmer, G. Diezemann, G. Hinze, and E. Rössler, Prog. Nucl. Magn. Reson. Spectrosc. 39, 191 (2001).

${ }^{28}$ E. R. deAzevedo, T. J. Bonagamba, and D. Reichert, Prog. Nucl. Magn. Reson. Spectrosc. 47, 137 (2005).

${ }^{29}$ M. Vogel, Phys. Rev. Lett. 101, 225701 (2008).

${ }^{30}$ S. A. Lusceac, M. R. Vogel, and C. R. Herbers, Biochim. Biophys. Acta, Proteins Proteomics 1804, 41 (2010).

${ }^{31}$ S. A. Lusceac and M. Vogel, J. Phys. Chem. B 114, 10209 (2010).

${ }^{32}$ K. Kämpf, B. Kremmling, and M. Vogel, Phys. Rev. E 89, 032710 (2014).

${ }^{33}$ S. A. Lusceac, M. Rosenstihl, M. Vogel, C. Gainaru, A. Fillmer, and R. Böhmer, J. Non-Cryst. Solids 357, 655 (2011).

${ }^{34}$ S. A. Lusceac, C. Gainaru, D. A. Ratzke, M. F. Graf, and M. Vogel, J. Phys. Chem. B 115, 11588 (2011).

${ }^{35}$ D. Sauer, B. Schuster, M. Rosenstihl, S. Schneider, V. Talluto, T. Walther, T. Blochowicz, B. Stühn, and M. Vogel, J. Chem. Phys. 140, 114503 (2014).

${ }^{36}$ M. Sattig, K. Elamin, M. Reuhl, J. Swenson, and M. Vogel, J. Phys. Chem. C 121, 6796 (2017).

${ }^{37}$ D. Demuth, M. Sattig, E. Steinrücken, M. Weigler, and M. Vogel, Z. Phys. Chem. 232, 1059 (2018)

${ }^{38}$ N. Bloembergen, E. M. Purcell, and R. V. Pound, Phys. Rev. 73, 679 (1948).

${ }^{39}$ A. Abragam, Principles of Nuclear Magnetism (Oxford University Press, 1983).

${ }^{40}$ W.-K. Rhim, A. Pines, and J. S. Waugh, Phys, Rev. B 3, 684 (1971).

${ }^{41}$ K. Takegoshi and C. A. McDowell, Chem. Phys. Lett. 116, 100 (1985)

${ }^{42}$ S. Sturniolo and K. Saalwächter, Chem. Phys. Lett. 516, 106 (2011).

${ }^{43}$ R. Fechete, D. E. Demco, and B. Blümich, J. Chem. Phys. 118, 2411 (2003).

${ }^{44}$ M. Sattig and M. Vogel, J. Phys. Chem. Lett. 5, 174 (201414).

${ }^{45}$ M. Sattig, S. Reutter, F. Fujara, M. Werner, G. Buntkowsky, and M. Vogel, Phys. Chem. Chem. Phys. 16, 19229 (2014).

${ }^{46}$ M. Weigler, M. Brodrecht, G. Buntkowsky, and M. Vogel, J. Phys. Chem. B 123, 2123 (2019).

${ }^{47}$ S. Maeda, K.-K. Kunimoto, C. Sasaki, A. Kuwae, and K. Hanai, J. Mol. Struct. 655, 149 (2003).

${ }^{48}$ D. R. S. Kushwaha, K. B. Mathur, and D. Balasubramanian, Biopolymers 19, 219 (1980).

${ }^{49}$ S. Matsui, Chem. Phys. Lett. 179, 187 (1991)

${ }^{50}$ S. Schneider and M. Vogel, J. Chem. Phys. 149, 104501 (2018).

${ }^{51}$ S. W. Englander, N. W. Downer, and H. Teitelbaum, Annu. Rev. Biochem. 41, 903 (1972).

${ }^{52}$ E. Liepinsh and G. Otting, Magn. Reson. Med. 35, 30 (1996).

${ }^{53}$ C. E. Dempsey, Prog. Nucl. Magn. Reson. Spectrosc. 39, 135 (2001).

${ }^{54}$ Y. Bai, J. S. Milne, L. Mayne, and S. W. Englander, Biochim. Biophys. Acta, Gen. Subj. 17, 75 (1993). 
${ }^{55}$ M. T. McMahon, A. A. Gilad, J. Zhou, P. Z. Sun, J. W. M. Bulte, and P. C. M. van Zijl, Magn. Reson. Med. 55, 836 (2006).

${ }^{56}$ W. E. Hull, H. R. Kricheldorf, and M. Fehrle, Biopolymers 17, 2427 (1978).

${ }^{57}$ T. Segawa, F. Kateb, L. Duma, G. Bodenhausen, and P. Pelupessy, ChemBioChem 9, 537 (2008).

${ }^{58}$ A. Esadze, D.-W. Li, T. Wang, R. Brüschweiler, and J. Iwahara, J. Am. Chem. Soc. 133, 909 (2011).

${ }^{59}$ Q. Z. Ni, E. Markhasin, T. V. Can, B. Corzilius, K. O. Tan, A. B. Barnes, E. Daviso, Y. Su, J. Herzfeld, and R. G. Griffin, J. Phys. Chem. B 121, 4997 (2017). ${ }^{60}$ J. Hirschinger, H. Miura, K. H. Gardner, and A. D. English, Macromolecules 23, $2153(1990)$
${ }^{61}$ J. W. Mack, M. G. Usha, J. Long, R. G. Griffin, and R. J. Wittebort, Biopolymers 53, 9 (2000).

${ }^{62}$ M. Vogel and E. Rössler, J. Phys. Chem. A 102, 2102 (1998).

${ }^{63}$ T. Blochowicz, C. Karle, A. Kudlik, P. Medick, I. Roggatz, M. Vogel, C. Tschirwitz, J. Wolber, J. Senker, and E. Rössler, J. Phys. Chem. B 103, 4032 (1999).

${ }^{64}$ M. Vogel, P. Medick, and E. A. Rössler, Annu. Rep. NMR Spectrosc. 56, 231 (2005).

${ }^{65}$ R. Kubo and K. Tomita, J. Phys. Soc. Jpn. 9, 888 (1954).

${ }^{66}$ H. A. Resing, J. Chem. Phys. 43, 669 (1965).

${ }^{67}$ B. Pötschner, F. Mohamed, A. Lichtinger, D. Bock, and E. A. Rössler, J. Chem. Phys. 143, 154506 (2015). 